\title{
Prévention de l'oxydation des acides gras dans un produit cosmétique : mécanismes, conséquences, moyens de mesure, quels antioxydants pour quelles applications?
}

\author{
Armelle JUDDE \\ ITERG, Rue Monge, \\ Parc Industriel Bersol 2, \\ 33600 Pessac \\ $<$ a.judde@iterg.com>
}

\begin{abstract}
To recall the general mechanism of fatty acid oxidation, a focus is made on the different degradation product families and their impact on the quality of blend or formulated fats and oils. Because of the importance of the analytical method choice to assess the oxidative status of the products, a review underlines the precautions required to interpret the results. Another important point concerns the Good Manufacturing Practices to reduce the oxidative risk, and the respective advantages and drawbacks of a selection of synthetic or natural antioxidants in cosmetic applications, conclude this short review.
\end{abstract}

Key words: chemical oxidation, fatty acid, hydroperoxides, oxidative product, antioxidants in cosmetic applications
L'objectif de cette présentation est de sensibiliser les utilisateurs de corps gras à l'intérêt d'intégrer le plus en amont possible la notion de risque oxydatif dans la gestion de ces matières premières et des formulations cosmétiques en contenant, en dressant un panorama général de la problématique de l'oxydation. La notion de corps gras recouvre les produits $d^{\prime}$ origine naturelle, en particulier les huiles végétales (principalement constituées d'acides gras), mais également les cires ou les phospholipides.

En effet, lorsqu'ils sont extraits de leur environnement protecteur naturel (cellules), tous les corps gras subissent au cours de leur conservation ou de leur utilisation des altérations oxydatives. Les principaux composés oxydables sont les acides gras insaturés, à l'état libre ou estérifiés en triglycérides, et porteurs d'un ou plusieurs site(s) réactionnel(s) que sont les doubles liaisons ; d'autres composés de nature lipidique sont par ailleurs oxydables: vitamines liposolubles, stérols... Le phénomène d'oxydation des acides gras conduit à une dégradation organoleptique de la matrice qui les contient, avec apparition d'une flaveur caractéristique « rance » qui modifie la qualité marchande du produit et conditionne directement sa durée de vie.

Pour mémoire, rappelons qu'un corps gras naturel organique type huile végétale peut subir trois grands types d'altération :

- oxydation : I'oxygène de l'air est dans ce cas le seul paramètre nécessaire et indispensable à l'initiation des réactions de dégradation;

- hydrolyse: chimique ou enzymatique qui conduit à la formation de glycérides partiels et d'acides gras libres (non abordé dans cet exposé):

- altération thermique: chauffage pour des températures supérieures à 100 voire $150^{\circ} \mathrm{C}$, conduit à la formation de polymères, de composés cycliques ou isomérisés (non abordé dans cet exposé).

\section{Les étapes de l'oxydation des acides gras}

Le rancissement oxydatif est un phénomène purement chimique et spontané dès lors que des acides gras insaturés (comportant au moins une double liaison) sont en présence d'oxygène atmosphérique ; notons à ce stade que la lumière ou la température sont des facteurs accélérateurs mais ne sont pas des éléments nécessaires et suffisants pour déclencher des phénomènes d'oxydation. Ce phénomène chimique se caractérise également par son caractère évolutif (dû à la succession, dans le temps, de différentes réactions chimiques, conduisant à plusieurs familles de produits réactionnels intermédiaires et finaux), irréversible et altératif (puisque l'attaque des acides gras par l'oxygène atmosphérique conduit à des dégradations organoleptiques et fonctionnelles, affectant directement la qualité marchande des corps gras et des produits finis).

Parmi les produits de dégradations, les composés aldéhydiques volatils jouent un rôle clef car ils sont responsables des flaveurs de " rance »: pour cette raison, la réaction d'oxydation est souvent associée à la notion de rancissement chimique aldéhydique (à distinguer du rancissement butyrique ou du rancissement cétonique, qui sont des phénomènes enzymatiques d'hydrolyse).

Plus précisément, la réaction d'oxydation est une réaction de type radicalaire: rappelons qu'un radical libre est une espèce chimique qui possède un électron non apparié dit « célibataire ", ce qui en fait un intermédiaire réactionnel instable à courte durée de vie, qui se stabi- lise en arrachant un électron à une autre espèce chimique, qui se trouve alors elle-même déstabilisée puisque porteuse d'un électron non apparié. Ceci permet de comprendre le caractère autocatalytique de la réaction d'oxydation, dont la cinétique suit une courbe exponentielle croissante, comportant trois phases distinctes :

- phase d'initiation : lente, elle correspond à la formation des radicaux libres ;

- phase de propagation: rapide, elle correspond à la formation, à partir des radicaux libres, des peroxydes et à l'apparition consécutive des composés secondaires d'oxydation ;

- phase de terminaison: elle correspond à la disparition des peroxydes, à l'accumulation des composés secondaires d'oxydation, c'est-àdire à l'oxydation complète du substrat. D'un point de vue pratique, dans les produits cosmétiques, les cinétiques des réactions (en regard des durées de vie des produits) atteignent rarement le stade de terminaison.

Comme le montre la figure 1, la phase de propagation peut elle-même être découpée en deux étapes séquentielles :

- La première étape correspond à l'apparition des peroxydes, composés primaires d'oxydation, à partir des radicaux libres instables: la quantité de peroxydes formés peut être évaluée analytiquement grâce à la détermination de l'indice de peroxyde. À ce stade, dit de peroxydation, la flaveur de rance peut ne pas être perceptible, la qualité marchande du produit non encore altérée ;

- La deuxième étape se traduit par l'évolution des hydroperoxydes en composés secondaires d'oxydation, par deux voies principales :

- une voie dite de scission, conduisant par coupure à la libération de composés volatils (chânes carbonées courtes et moyennes), notamment aldéhydiques, responsables des flaveurs 


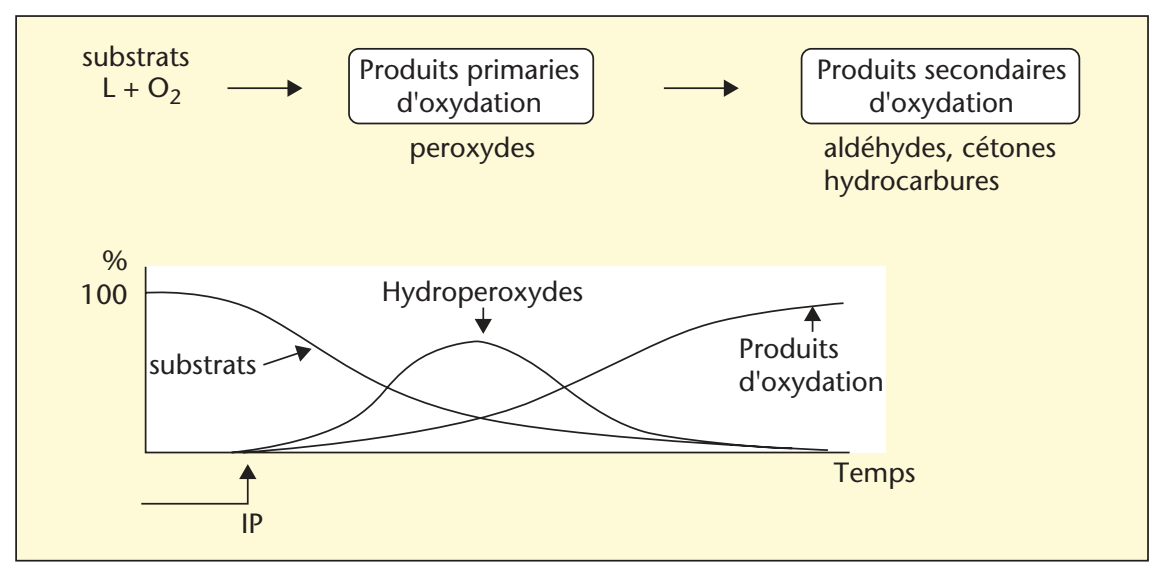

Figure 1. Représentation simplifiée de la cinétique de formation et de décomposition des hydroperoxydes et de la cinétique de formation des produits secondaires d'oxydation.

de rance, caractérisés par un seuil de détection très faible ;

- une voie dite de remaniement, conduisant suite à différents types de pontage intra- ou inter-acides gras ou suite à l'apparition de fonctions oxydées (fonctions cétone, époxy, hydroxy) sur les acides gras, à la formation d'acides gras oxydés, de triglycérides oxydés, de polymères de triglycérides oxydés. À ce stade dit de rancissement, la flaveur de rance est bien entendu perceptible, et peut être accompagnée par d'autres conséquences $d^{\prime}$ 'ordre fonctionnel (aspect, couleur, texture) et d'ordre physiologique (altérations des acides gras essentiels et des vitamines liposolubles). L'examen de la formation des radicaux et hydroperoxydes d'acides gras, en conditions d'autoxydation (en présence d'oxygène, en absence de lumière) illustre la complexité de la réaction d'oxydation. Suite à un apport énergétique, I'hydrogène situé en $\alpha$ (position allylique) de chaque côté de la double liaison de I'acide gras subit une coupure homolytique; cet hydrogène est en effet le plus labile car il subit l'effet électrodonneur de la double liaison voisine. Apparaît alors un radical d'acide gras, sur lequel la double liaison peut migrer (isomérie de position) et/ou s'isomériser (isomérie géométrique cis/trans) : on parle de métamérie. L'oxygène atmosphérique peut alors se fixer sur le radical $d^{\prime}$ acide gras pour former un radical peroxydique, instable, qui se stabilise sous forme d'un hydroperoxyde d'acide gras, grâce à un $\mathrm{H}$ arraché en $\alpha$ de la double liaison d'un autre acide gras. Ainsi, la figure 2 illustre le cas d'un acide gras porteur d'une double liaison, à partir duquel se forment quatre hydroperoxydes isomères de position, avec chacun deux isomères géométriques potentiels. Moyennant quoi, le pool d'hydroperoxydes qui se forme dans un produit formulé comportant une ou plusieurs matière(s) grasse(s) comportant chacune plusieurs acides gras, est très complexe, et contribue à la formation libres (acidité oléique faible), ne comportant pas de traces de métaux pro-oxydants, contribuera à améliorer la stabilité à l'oxydation de la formule.

Quelques mots sur le cas particulier de l'oxydation en présence de lumière, pour lequel deux situations peuvent se présenter :

- Photoxydation directe : la lumière joue le rôle d'accélérateur des cinétiques des réactions d'oxydation, les mécanismes chimiques restent les mêmes;

- Oxydation photosensiblisée: grâce à la présence nécessaire d'un agent photosensiblisateur (pigments type chlorophylle, certains colorants, certaines vitamines), l'oxygène de l'air est activé, passant de son état fondamental dit " triplet » à un état excité dit "singulet», état dans lequel l'oxygène a suffisamment d'énergie pour se fixer directement sur l'acide gras (par un mécanisme dit de «ène addition »), sans passer par l'étape radicalaire. Les mécanismes réactionnels sont donc différents, les produits formés sont différents.

Dans le cas d'un produit cosmétique formulé, il est nécessaire de considérer la nature du corps gras dans son environnement, en s'intéressant plus particulièrement aux liaisons qu'il a pu engager avec d'autres constituants (protéines), ou à sa répartition physique dans la formule (phase continue ou émulsion eau/huile ou émulsion huile/eau) : ces éléments conditionnent en effet l'accessibilité des acides gras à l'oxygène de l'air ou à l'oxygène dissous. De ce fait, la transposition directe des résultats d'un test de stabilité conduit sur un corps gras, au produit formulé, n'est pas envisageable.

En effet, les constituants suivants d'une formule sont susceptibles d'interagir avec le corps gras et $d^{\prime}$ influencer le déroulement des réactions d'oxydation :

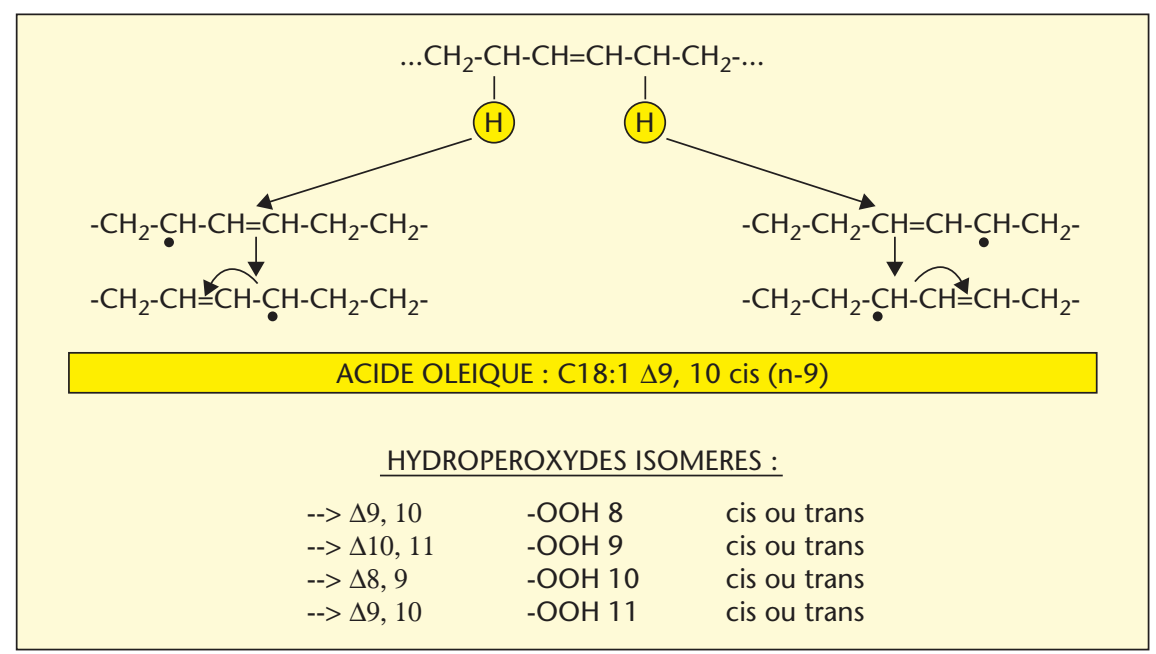

Figure 2. Cas de la formation des hydroperoxydes isomères à partir d'un acide gras mono-insaturé. 
- eau : en présence d'eau, les risques d'hydrolyse augmentent ; or, plusieurs études font état de l'oxydabilité supérieure des acides gras lorsqu'ils sont à l'état libre ;

- traces de métaux pro-oxydants (fer et cuivre sous forme libre) : ils augmentent les cinétiques de formation des radicaux et de décomposition des hydroperoxydes pour des teneurs faibles (centaines de $\mathrm{ppb}$ ) ;

- protéines, peptides, acides aminés: de manière générale, il est noté un rôle favorable des fonctions amines sur la stabilité oxydative des huiles végétales :

- émulsifiants: placés aux interfaces, ils sont souvent associés à une limitation de la diffusion de l'oxygène entre les phases aqueuses et huileuses des émulsions.

Les réactions d'oxydation conduisent finalement à l'apparition de divers composés « primaires » et « secondaires » d'oxydation dont on ne connaît pas les actions physiologiques ou la toxicité chez l'homme. Les études conduites chez l'animal apportent cependant des informations qui permettent d'apprécier les effets potentiels respectifs des différents produits d'oxydation :

- Les effets physiologiques des acides gras peroxydés en test de toxicité aiguë comprennent perte de poids, retard de croissance, mort, irritations intestinales, diarrhées, dilatation du foie et des reins, difficultés respiratoires, réactions inflammatoires, formation de tumeurs, augmentation du risque cardio-vasculaire et lésions placentaires. Cependant, compte tenu des doses de peroxydes mises en contact par voie topique dans le cadre de l'application de produits cosmétiques, il existe une marge de sécurité très importante au regard de la $\mathrm{DL}_{50}$

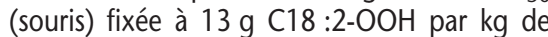
poids corporel. Par ailleurs, les effets sont atténués s'il y a une prise conjointe de vitamine $\mathrm{E}$; - II n'existe pas de données épidémiologiques concernant l'effet à long terme d'une consommation régulière de matières grasses peroxydées (toxicité chronique) ;

- En ce qui concerne les autres produits de dégradation, les composés les plus réactifs sont les aldéhydes (dont le MDA), qui peuvent se fixer sur les protéines et l'ADN (démontré chez I'animal), mais il n'existe pas de données chez I'homme.

\section{Les méthodes de mesure de l'oxydation et du rancissement}

Face à une problématique d'évaluation du niveau d'oxydation d'un corps gras, plusieurs approches sont envisageables :

- soit mesurer la disparition des substrats de la réaction d'oxydation c'est-à-dire suivre la teneur en acides gras au cours du temps par chromatographie en phase gazeuse (CPG) ; - soit mesurer la consommation en réactif de la réaction d'oxydation c'est-à-dire suivre la diminution de la pression partielle en oxygène ; - soit suivre l'apparition des produits primaires d'oxydation c'est-à-dire les hydroperoxydes ; - soit suivre l'apparition des produits secondaires d'oxydation, c'est-à-dire les composés volatils, les aldéhydes volatils ou non, les composés oxydés et/ou polymérisés non volatils ;

Compte tenu des faibles quantités de produits d'oxydation formés (de l'ordre des ppb ou ppm pour les composés volatils, de l'ordre des ppm ou $0,1 \%$ pour les hydroperoxydes), les méthodes sélectionnées doivent êtres sensibles et spécifiques. Pour ces raisons, les deux premières approches présentées sont peu couramment retenues.

\section{Préliminaire à la mesure de l'oxydation}

En préalable à l'évaluation du niveau d'oxydation d'un corps gras formulé, il est nécessaire de l'extraire de la matrice, de la manière la plus quantitative, sélective et non altérante qu'il soit. Cette opération d'extraction impose la mise en œuvre d'un certain nombre de précautions afin de ne pas induire de stress oxydatif. Ainsi, la plupart des méthodes dites d'extraction, dont la finalité n'est pas d'obtenir un extrait mais de quantifier la teneur en matière grasse d'un produit, sont généralement à prohiber. C'est par exemple le cas des méthodes par reflux à chaud (type méthode Soxhlet Hexane), souvent utilisées pour des matrices pulvérulentes, des graines, et des méthodes comportant une hydrolyse acide ou alcaline, plutôt réservées au traitement des produits complexes de l'agroalimentaire (produits laitiers, carnés, céréaliers...). Par contre, les méthodes mettant en jeu une combinaison de solvants polaires et apolaires, à froid, sont tout à fait indiquées dans le cas d'une étude d'évaluation du niveau d'oxydation: citons par exemple la méthode de Folch (mélange chloroforme/méthanol) et la méthode normalisée NF V03-030 (hexane/isopropanol), qui permettent l'obtention d'un extrait quantitatif et qualitatif, sur lequel les méthodes de mesure de l'oxydation et du rancissement peuvent être appliquées.

\section{Mesure des composés primaires d'oxydation}

L'indice de peroxyde est la méthode incontournable : bien qu'imparfaite puisqu'en lien avec la peroxydation et non le rancissement, cette méthode normalisée (NF T60-220 annulée et remplacée par ISO 3960), avec une bonne répétabilité, dispose par ailleurs de valeurs de référence (Codex alimentarius, Pharmacopée), et figure quasi systématiquement dans les cahiers des charges « corps gras » avec classiquement une valeur seuil fixée à 10 méq $\mathrm{O}_{2}$ par $\mathrm{kg}$ de matière pour une huile raffinée.

Cette mesure peut être remplacée par une détermination de l'absorbance UV à 232 nm, normalisée (norme NF EN ISO 3656), corrélée à la présence de formes diènes conjuguées, qui apparaissent sur les acides gras comportant au moins deux doubles liaisons, consécutivement au phénomène de métamérie lors de la formation des peroxydes: très facile à mettre en œuvre, cette détermination présente l'inconvénient d'être moins sensible, et de ne pas être liée à des valeurs de référence, donc difficile d'interprétation dans l'absolu. Cependant, ce type de suivi présente un intérêt dans le cadre d'un contrôle qualité pour une source de corps gras donnée.

\section{Mesure des composés secondaires d'oxydation}

Un certain nombre de méthodes spectrophotométriques sont disponibles, toutes sont basées sur la mesure de l'intensité de la coloration résultant de la complexation d'une famille de produits secondaires de dégradation oxydative avec un réactif spécifique. Les deux méthodes suivantes sont le plus souvent pratiquées : - indice de para-anisidine (NF EN ISO 6885), plus spécifique des aldéhydes conjugués ; - test TBA (IUPAC 2.531) ou test à l'acide thiobarbiturique, plus spécifique des formes aldéhydiques apparaissant à partir d'acides gras comportant au moins trois doubles liaisons;

Ces méthodes sont très spécifiques d'une famille de produits de dégradation oxydative, et sont par ailleurs sujettes à de nombreux risques d'interférences avec des constituants d'autres natures susceptibles de se complexer avec le réactif de départ. Leur utilisation et leur interprétation doivent être éclairées par des éléments de formulation et par l'expérience de I'utilisateur.

Les méthodes chromatographiques (associant la CPG à la technique de l'espace de tête statique ou dynamique) présentent l'avantage d'être représentatives des composés volatils directement responsables du caractère rance. Lorsqu'elles sont mises en œuvre avec les précautions techniques requises, elles constituent un outil pertinent, notamment pour le suivi de l'oxydation au cours du temps.

Le choix des méthodes doit s'effectuer au cas par cas, en fonction du modèle étudié. Le plan expérimental doit toujours comporter un produit témoin ou un produit de référence et repose souvent sur la combinaison de plusieurs méthodes complémentaires, généralement une méthode ciblant les hydroperoxydes et une méthode ciblant les composés secondaires $d^{\prime}$ oxydation. Rappelons enfin que l'outil le plus sensible pour détecter une flaveur rance reste le 
nez et que, de ce fait, un contrôle olfactif des corps gras, même s'il n'est pas quantitatif, trouve tout à fait sa place dans un laboratoire de contrôle.

\section{Les familles d'antioxydants}

Lors de la confrontation à une problématique d'oxydation, l'ambition la plus raisonnable consiste à retarder l'apparition des premières réactions d'oxydation (c'est-à-dire allonger la phase d'initiation) mais en aucun cas à supprimer la réaction d'oxydation, ce qui ne pourrait se concevoir qu'en absence totale d'oxygène. Les bonnes pratiques de fabrication apportent plusieurs possibilités d'actions en ce sens : - pour minimiser l'apport énergétique (température, lumière) en optimisant les conditions de stockage des matières premières et de conditionnement des produits finis, en maîtrisant les étapes du procédé comportant un chauffage : - pour réduire le contact avec l'oxygène, en optimisant les étapes du procédé comportant une agitation, et en sélectionnant des emballages modifiés « actifs »;

- pour réduire la présence de métaux prooxydants, en introduisant cette spécification dans les cahiers des charges « matières premières » et en veillant à identifier les étapes du procédé impliquant un matériel non inoxydable ;

- pour enfin, antioxyder le corps gras dans des conditions d'efficacité optimales.

Pour ce faire, différentes catégories d'antioxydants sont disponibles:

- les antioxydants primaires phénoliques de rupture de chaîne: ils s'oxydent à la place des acides gras, puis, contrairement à ces derniers, ont la propriété de se stabiliser sous une forme qui n'évolue pas vers le stade radicalaire. Ces molécules ont toutes dans leur structure une fonction de type $\mathrm{X}-\mathrm{H}$ avec un hydrogène $\mathrm{H}$ labile :

Dans cette catégorie, on retrouve les antioxydants de synthèse classiques BHT (E321) et BHA (E320) qui présentent une très bonne liposolubilité, une excellente efficacité dans les huiles végétales, mais qui peuvent être à l'origine de colorations jaunes dans les formules blanches ; leur utilisation est aujourd'hui réservée à quelques rares applications du fait de la remise en question de leur totale innocuité. Les gallates (E310 à E312), esters de I'acide gallique, sont quant à eux très efficaces car chaque molécule de gallate est porteuse de trois fonctions hydroxy avec un $\mathrm{H}$ labile, mais présentent l'inconvénient d'être peu liposolubles (la meilleure liposolubilité étant atteinte avec le gallate de dodécyl). Par ailleurs, lorsqu'ils sont utilisés en formulation en présence d'eau et de traces métalliques de fer, ils engendrent une réaction colorée bleue-noire, qu'il est cepen- dant possible d'éliminer en ajoutant dans la formule un agent chélateur de métaux, type acide citrique.

Cette catégorie comprend également les antioxydants naturels, parmi lesquels les tocophérols (E306 à E309) sont les plus connus, et très couramment utilisés du fait de leur liposolubilité, de leur synergie avec d'autres molécules antioxydantes et de la possibilité de les utiliser « quatum satis ». En matière d'antioxydation, les formes $\gamma$ et $\delta$ tocophérols offrent la meilleure efficacité, suivie par les formes $\alpha$ et $\beta$ tocophérols. L'a tocophérol est la forme $\mathrm{d}^{\prime}$ apport vitaminique (vitamine E) privilégiée : dans ce cas, l'apport peut se faire sous forme libre ou estérifiée. Par contre, lorsque c'est l'effet antioxydant qui est recherché, les tocophérols doivent être impérativement formulés sous forme libre, de telle sorte que la fonction hydroxy ne soit pas engagée dans une liaison ester.

Parmi les antioxydants naturels, le marché propose de nombreux extraits végétaux riches en molécules phénoliques ayant une activité de rupture de chaîne (extraits de romarin, extraits de thé vert, extraits de raisin...) dont le statut réglementaire n'est pas clarifié à ce jour en France.

- Les antioxydants secondaires synergistes, prolongeant la durée de vie des antioxydants primaires: c'est le cas de l'acide ascorbique, hydrosoluble, et de son homologue plus liposoluble (jusqu'à 250-300 ppm), le palmitate d'ascorbyle.

- Les antioxydants secondaires chélateurs de métaux, piégeant les métaux prooxydants (fer et cuivre) : c'est le cas de l'acide citrique ou des lécithines, qui présentent une efficacité pour des doses d'incorporation faibles (à partir de 50 ppm).

- Les antioxydants secondaires à rôle spécifique, agissant sur l'oxygène : ainsi, le $\beta$-carotène est capable de piéger l'oxygène sous sa forme singulet, et peut donc intervenir dans le cas d'une oxydation photosensibilisée. Cependant, cet antioxydant reste difficile à mettre en œuvre ; son efficacité étant étroitement liée aux conditions opératoires et à la nature de la matrice.

Signalons également l'effet antioxydant très intéressant des phospholipides (lécithines) utilisés en synergies avec les tocophérols naturellement présents dans les huiles végétales, bien connu empiriquement depuis de nombreuses années, et démontré par des données objectivées dans une étude récente [1].

De manière générale, il est tout à fait pertinent de combiner les antioxydants entre eux, en associant par exemple, l'efficacité d'un antioxydant primaire phénolique avec un antioxydant secondaire synergiste, prolongeant la durée de vie des premiers, et un antioxydant secondaire chélateur de métaux. Les produits disponibles sur le marché valorisent largement les propriétés de synergies et offrent par ailleurs un confort d'utilisation en déclinant des formes hydrosolubles, liposolubles, désodorisées, décolorées, liquides, pulvérulentes... Les facteurs limitants, dans le cas d'application en formulation cosmétiques, restent souvent la couleur et l'odeur résiduelles.

Le choix d'un antioxydant implique de considérer plusieurs éléments :

- La réglementation et le domaine d'application: en l'absence de données dans le secteur cosmétique, référence est souvent faite au domaine de l'agroalimentaire, pour lequel il existe des valeurs limites pour l'utilisation des antioxydants de synthèse (BHT, BHA et gallates) pour des applications spécifiques, et la recommandation "quantum satis" pour les tocophérols, l'acide ascorbique et l'acide citrique.

- La solubilité dans la formule : il est intéressant de souligner l'approche dite du paradoxe polaire, défendue par le Pr Edwin Frankel, qui consiste à privilégier les antioxydants de nature hydrophile dans les bases $100 \%$ huile et, a contrario, de privilégier les antioxydants de nature lipophile dans les émulsions huile/eau. Dans les deux cas, l'action des antioxydants vise en priorité les interfaces, de manière à favoriser leur concentration.

- La stabilité thermique et la résistance aux étapes du procédé de fabrication.

\section{Les études d'efficacité des antioxydants}

Pour mesurer l'efficacité d'un antioxydant, deux possibilités s'offrent au formulateur :

- Soit mettre en œuvre un test de vieillissement en conditions normales de stockage : parfaitement représentatif, ce type de test est long et incompatible avec les contraintes de développement d'un produit.

- Soit mettre en œuvre un test accéléré, pour « gagner » du temps lors des développements de produits. Plusieurs types de tests accélérés peuvent être envisagés :

- des tests très modélisés, mettant en œuvre des substrats modèles (esters) dans des milieux modèles parfois éloignés de la matrice « huile», et dans des conditions souvent assez drastiques. Citons à titre d'exemples le test DPPH (dipicrylphénylhydrazine), le test de cooxydation au $\beta$-carotène, et plus récemment mis au point, le test ORAC (Oxygen Radical Absorbance (apacity). Le principe de ce dernier est le suivant : mesure de la destruction d'une protéine végétale, qui possède la propriété de fluorescer lorsqu'elle est soumise à un rayonnement lumineux spécifique. Sous l'action des radicaux libres présents dans le milieu réaction- 
nel, la protéine est détruite et perd sa fluorescence, tandis qu'en présence d'un capteur de radicaux libres (antioxydant) la fluorescence persiste : ce test permet d'évaluer la capacité globale d'antioxydation (pouvoir antiradicalaire) d'un extrait végétal, par référence à un standard qui est le TROLOX (vitamine E sous forme hydrosoluble, dépourvue de chaîne carbonée). Ces tests modélisés permettent de réaliser un premier screening conduisant au classement des antioxydants testés en deux groupes : ceux qui ont une efficacité, ceux qui en ont aucune ;

- des tests d'oxydabilité accélérée types test Rancimat (ISO 6886), Oxydograph ou Oxipres. Le test Rancimat est le plus connu : la spécification de TIR (soit le Temps d'Induction au test Rancimat, exprimé en heures) est encore utilisée dans les cahiers des charges des corps gras. Elle correspond au temps pendant lequel I'huile végétale a résisté à un stress oxydatif. Le principe est le suivant : la prise d'essai de corps gras est soumise à une température élevée $\left(98^{\circ} \mathrm{C}\right)$ et à un bullage intensif d'air, ce qui déclenche les réactions d'oxydation, et notamment la libération d'acides organiques volatils, entraînés et piégés dans une cellule contenant de l'eau distillée, dont la conductivité va varier consécutivement. L'enregistrement repère le moment à partir duquel cette conductivité varie et qui correspond au TIR. Une application du test Rancimat à la comparaison de l'efficacité de plusieurs systèmes antioxydants est présentée en figure 3.

L'avantage de ce type de test est la possibilité de suivre en parallèle plusieurs échantillons, avec des durées d'analyse réduites. L'inconvénient majeur repose sur les conditions mêmes du test, très drastiques, et peu représentatives des conditions normales de stockage. En conséquence, les résultats obtenus grâce à un

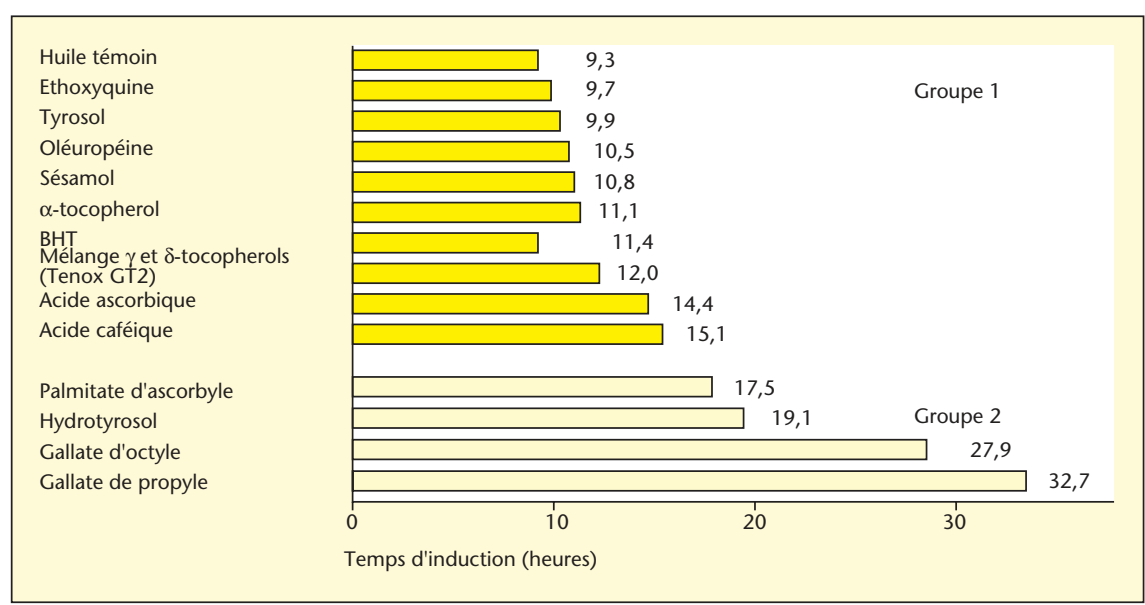

Figure 3. Exemple d'application du test Rancimat $\left(98^{\circ} \mathrm{C}\right)$ au classement de l'efficacité antioxydante de plusieurs molécules (dose : 400 ppm) sur une huile végétale [2].

test Rancimat ne peuvent être transposés directement ;

- les tests en conditions accélérées en enceinte thermostatée: les conditions de ces tests doivent être ajustées au cas pas cas, à l'objectif de l'essai, à la nature de la matrice, à la nature du corps gras, et débouchent sur le choix des facteurs accélérateurs de l'oxydation (niveau de température, présence de la lumière), sur la sélection des méthodes d'évaluation de l'oxydation et sur la définition des points de cinétique. Dans tous les cas, les conditions seront choisies les plus douces possible, ce qui implique des tests assez lents (en semaines en général), mais qui réservent l'approche la plus cohérente avec ce qui se passe en conditions normales de stockage.

\section{Conclusions}

Les phénomènes d'oxydation des acides gras sont complexes et difficiles à prévoir. Cepen- dant, la connaissance des propriétés des corps gras et la maîtrise du procédé et de l'environnement général, permettent $d^{\prime}$ agir, le plus en amont possible, pour retarder dans le temps l'apparition des premiers signes de l'oxydation. Les conséquences de l'oxydation d'un corps gras dans une formule cosmétique sont essentiellement d'ordre fonctionnel (aspect, texture), nutritionnel (acides gras polyinsaturés, vitamines) et sensoriel (odeur, couleur, dans ment la qualité marchande du produit.

\section{RÉFÉRENCES}

1. JUDDE A, VILLENEUVE $P$, et al. Antioxidant effect of soy lecithins on vegetable oil stability and their synergism with tocopherols. JAOCS, 2003 ; vol. 80, nº 11.

2. ROSSIGNOL CASTERA A, BOSQUE F. Nouvelle approche des antioxydants. OCL 1994 ; vol. 1, $n^{\circ} 2$. certains cas le goût): elles affectent directe- 